\begin{tabular}{|c|l|}
\hline Title & Crossover between Lévy and Gaussian regimes in first-passage processes \\
\hline Author(s) & Inoue, Jun-ichi; Sazuka, Naoya \\
\hline Citation & $\begin{array}{l}\text { Physical Review E, 76(2), 0211111 } \\
\text { https://doi.org/10.1103/PhysRevE.76.021111 }\end{array}$ \\
\hline Issue Date & 2007-08-17 \\
\hline Doc URL & http://hdl.handle.net/2115/28043 \\
\hline Rights & Copyright $\odot 2007$ A merican Physical Society \\
\hline Type & article \\
\hline File Information & PRE76.pdf \\
\hline
\end{tabular}

Instructions for use 


\title{
Crossover between Lévy and Gaussian regimes in first-passage processes
}

\author{
Jun-ichi Inoue ${ }^{1, *}$ and Naoya Sazuka ${ }^{2, \dagger}$ \\ ${ }^{1}$ Complex Systems Engineering, Graduate School of Information Science and Technology, Hokkaido University, N14-W9, Kita-ku, \\ Sapporo 060-0814, Japan \\ ${ }^{2}$ Sony Corporation, 1-7-1 Konan Minato-ku, Tokyo 108-0075, Japan
}

(Received 15 August 2006; revised manuscript received 26 April 2007; published 17 August 2007)

\begin{abstract}
We propose an approach to the problem of the first-passage time. Our method is applicable not only to the Wiener process but also to the non-Gaussian Lévy flights or to more complicated stochastic processes whose distributions are stable. To show the usefulness of the method, we particularly focus on the first-passage time problems in the truncated Lévy flights (the so-called KoBoL processes from Koponen, Boyarchenko, and Levendorskii), in which the arbitrarily large tail of the Lévy distribution is cut off. We find that the asymptotic scaling law of the first-passage time $t$ distribution changes from $t^{-(\alpha+1) / \alpha}$-law (non-Gaussian Lévy regime) to $t^{-3 / 2}$-law (Gaussian regime) at the crossover point. This result means that an ultraslow convergence from the non-Gaussian Lévy regime to the Gaussian regime is observed not only in the distribution of the real time step for the truncated Lévy flight but also in the first-passage time distribution of the flight. The nature of the crossover in the scaling laws and the scaling relation on the crossover point with respect to the effective cutoff length of the Lévy distribution are discussed.
\end{abstract}

DOI: 10.1103/PhysRevE.76.021111

PACS number(s): 02.50.Ga, 02.50.Ey, 89.65.Gh

\section{INTRODUCTION}

The first-passage process or the first-passage time (FPT) problem deals with the event where a diffusing particle or a random-walker first reaches a specific site at a specific time [1]. These FPT problems have been studied in various research fields, such as statistical physics, chemistry [2], and biological neuroscience $[3,4]$. In finance, several authors [5-12] have analyzed tick-by-tick data of the US dollar/Japanese yen (USD/JPY) exchange rate and studied the FPT distribution for which the FPT is defined by the time that the rate first moves out from a given range.

Among these studies, the USD/JPY exchange rates of the Sony Bank [13] are reproduced from the market rates by using some rate windows with a width of 0.1 yen $[11,12]$. That is, if the USD/JPY market rate changes by more than 0.1 yen, the Sony Bank rate for USD/JPY is updated to the market rate; otherwise it remains constant. In this sense, it is possible for us to say that the procedure for determining the USD/JPY exchange rate of the Sony Bank is essentially the first passage process. Despite many demands from various research fields and business in financial markets, one could obtain explicit analytical expressions or solutions of the FPT distribution only in very few cases. In addition, except for a few cases [14], most of the analytical expressions are of the ordinary Wiener process (ordinary Brownian motion).

Based on this fact, here we propose an approach to the problem of the FPT or first-passage processes. Our method is applicable not only to the Wiener process but also to the anomalous diffusion of the non-Gaussian Lévy flights or more complicated stochastic processes. To show the usefulness of our approach, we particularly focus on the FPT problems in the truncated Lévy flights [15-17], in which the ar-

\footnotetext{
*j_inoue@ complex.eng.hokudai.ac.jp

†naoya.sazuka@jp.sony.com
}

bitrarily large tail of the Lévy distribution is cut off. Using the method, we find that the asymptotic scaling law of the FPT $t$ distribution changes from a $t^{-(\alpha+1) / \alpha}$-law (nonGaussian Lévy regime) to a $t^{-3 / 2}$-law (Gaussian regime) at some crossover point. This fact means that the crossover between non-Gaussian Lévy and Gaussian regimes is observed not only in the distribution of the real-time step of the truncated Lévy flight, which was reported by Mantegna and Stanley [15], but also in the FPT distribution of the flight. Moreover, we give a scaling relation on the crossover point with respect to the effective cutoff length of the Lévy distribution. The scaling relation enables us to predict the crossover point of the FPT distribution for a given truncated Lévy flight.

This paper is organized as follows. In the next section, we explain general formalism of our method and apply it to the FPT problem for the Wiener process, for which the solution of the FPT distribution is well-known, in order to check the validity of our method. In Sec. III, we show that our method is widely useful for a class of stable stochastic processes. We derive the FPT distribution for Lévy flight that includes Gaussian and Lorentzian stochastic processes as its special cases. For each stable stochastic process, we discuss the scaling law of the FPT distribution in the asymptotic regime. The analytical results are confirmed by computer simulations. In Sec. IV, we apply our method to the FPT problem of the truncated Lévy flight and discuss the crossover in the scaling laws of the FPT distribution between non-Gaussian Lévy and Gaussian regimes. The last section is a summary.

\section{GENERAL FORMALISM}

The problem we deal with in this paper is defined as follows. Let us consider the stochastic process $X_{k}: k$ $=0,1, \ldots, T$. For this time series, the FPT $t$ is defined by $t$ $=\min \left\{k \geqslant k_{0} ; X_{k}= \pm \epsilon\right\}$. Then, our problem is to obtain the distribution of $t$, namely, the first-passage time distribution $P(t)$. 
In other words, we evaluate the distribution of $t$, that is $P(t)$, which is defined as the survival probability that the time series $X_{k}$, starting from $k=k_{0}$, keeps staying within the range $\left[X_{k_{0}}-\epsilon, X_{k_{0}}+\epsilon\right]$ up to the time step $k_{0}+t$. The problem we are dealing with is motivated by the real mechanism of the Sony Bank foreign exchange rate $[11,12]$. The Sony Bank rate is the foreign exchange rate that the Sony Bank offers with reference to the market rate. Basically, trades can be made on the web [13] while the market is open. The Sony Bank rate depends on the market rate but is independent of the customers' orders. If the USD/JPY market rate changes by $\epsilon$ $= \pm 0.1$ yen or more, the Sony Bank rate for USD/JPY is updated to the market rate. For instance, for the stochastic process of the real market (what we call tick-by-tick data) $X_{0}, X_{1}, X_{2}, \ldots, X_{T}$ with $\left|X_{1}\right|<\epsilon$ and $\left|X_{2}\right|>\epsilon$, the Sony Bank rate stays flat from the time $k=0$ to $k=1$, as the market rate is in the range of $\epsilon= \pm 0.1$ yen based on the market rate at $k$ $=0$. When the market rate exceeds the range of $\epsilon=0.1$ yen at $k=2$, the Sony Bank rate is updated to the market rate. Obviously, the time interval $t=2-0=2$ here corresponds to the FPT we explained above and it is worthwhile for us to evaluate its distribution $P(t)$ to investigate statistical property of the Sony Bank USD/JPY rates.

To calculate the FPT distribution $P(t)$ for the time series $X_{k}$, we define the probability $P(1)$, which means the probability of the FPT is $t=1$ as

$$
P(1)=\lim _{T \rightarrow \infty} \frac{1}{T} \sum_{k=1}^{T} \Theta\left(\left|X_{k+1}-X_{k}\right|-\epsilon\right),
$$

where $\Theta(x)$ means the Heviside step function, namely, $\Theta(x)=1$ for $x \geqslant 0$ and $\Theta(x)=0$ for $x<0$. We usually solve a kind of (fractal) Fokker-Plank equation under some appropriate boundary conditions $[2-4,14,18,19]$ or use the so-called image method $[1,20]$ to discuss the FPT problem. However, as we saw in Eq. (1), our approach is completely different from such standard treatments. To evaluate the FPT (probability) distribution, say $P(1)$, we directly count the number of $t=1$, namely, $\mathcal{N}_{1}=\sum_{k=1}^{T} \Theta\left(\left|X_{k+1}-X_{k}\right|-\epsilon\right)$ appearing within quite a long time interval $T$. We might choose $T$ as a time interval during which the market is open. Then the ratio $\mathcal{N}_{1} / T$ should be expected to converge to $P(1)$ as $T$ tends to infinity. This is the meaning of Eq. (1) and is our basic idea for evaluating the FPT distribution. From our method, to evaluate the FPT distribution by counting $\mathcal{N}_{t}(t=1,2, \ldots)$, the probability $P(2)$ is also given by $\lim _{T \rightarrow \infty}\left(\mathcal{N}_{2} / T\right)$, that is to say,

$$
\begin{aligned}
P(2)= & \lim _{T \rightarrow \infty} \frac{1}{T} \sum_{k=1}^{T} \Theta\left(\left|X_{k+2}-X_{k}\right|-\epsilon\right)-P(1) \\
= & \lim _{T \rightarrow \infty} \frac{1}{T} \sum_{k=1}^{T} \Theta\left(\left|X_{k+2}-X_{k}\right|-\epsilon\right) \\
& -\lim _{T \rightarrow \infty} \frac{1}{T} \sum_{k=1}^{T} \Theta\left(\left|X_{k+1}-X_{k}\right|-\epsilon\right) .
\end{aligned}
$$

In the same way as the probability $P(2)$, the probability $P(3)$ is obtained as

$$
\begin{aligned}
P(3)= & \lim _{T \rightarrow \infty} \frac{1}{T} \sum_{k=1}^{T} \Theta\left(\left|X_{k+3}-X_{k}\right|-\epsilon\right)-P(1)-P(2) \\
= & \lim _{T \rightarrow \infty} \frac{1}{T} \sum_{k=1}^{T} \Theta\left(\left|X_{k+3}-X_{k}\right|-\epsilon\right) \\
& -\lim _{T \rightarrow \infty} \frac{1}{T} \sum_{k=1}^{T} \Theta\left(\left|X_{k+2}-X_{k}\right|-\epsilon\right) .
\end{aligned}
$$

We should notice that the probability $P(1)$ was canceled in expression (3). Thus we easily generalize this kind of calculation to evaluate the distribution $P(t)$ by repeating the above procedure as follows.

$$
\begin{aligned}
P(t)= & \lim _{T \rightarrow \infty} \frac{1}{T} \sum_{k=1}^{T} \Theta\left(\left|X_{k+t}-X_{k}\right|-\epsilon\right) \\
& -\lim _{T \rightarrow \infty} \frac{1}{T} \sum_{k=1}^{T} \Theta\left(\left|X_{k+t-1}-X_{k}\right|-\epsilon\right),
\end{aligned}
$$

where $P(1), \ldots, P(t-2)$ were all canceled in this final formula (4). Equation (4) is the starting point of our evaluation. At a glance, this equation seems to be just a definition of the FPT distribution; however, for some classes of stochastic processes, we can derive the explicit form of the FPT distribution from this simple equation. In the next section, we derive the FPT distribution for the Wiener process as a simple test of our method. We stress that our approach helps as an intuitive account for the first-passage process and derivation of its distribution.

\section{Simple test of the method for Wiener stochastic processes}

To show the validity and usefulness of our method, we derive the FPT distribution from the above expression (4) for Wiener stochastic processes (Brownian motion). The ordinary Wiener process is described by $X_{t+1}=X_{t}+Y_{t}$, where the noise term $Y_{t}$ obeys the white Gaussian with zero mean and variance $\sigma^{2}$. Then we should notice that the difference $S_{t}$ $\equiv X_{k+t}-X_{k}$ is rewritten in terms of the sum of the noise terms $Y_{t}$ as $S_{t}=\sum_{j=0}^{t-1} Y_{k+j}$. As is well known, as the Gaussian process is stable, $S_{t}$ obeys the Gaussian with zero mean and $\left\langle\left(S_{t}\right)^{2}\right\rangle$ $=t \sigma^{2}$ variance. Using the same argument as $S_{t}, S_{t-1}$ also obeys the Gaussian with zero mean and $\left\langle\left(S_{t-1}\right)^{2}\right\rangle=(t-1) \sigma^{2}$ variance. Therefore the FPT distribution $P(t)$ derived by Eq. (4) leads to

$$
\begin{gathered}
P(t)=\Omega\left(p: S_{t}, S_{t-1}\right) \\
\Omega(p: l, m) \equiv \int_{-\infty}^{\infty} p(l) \Theta(|l|-\epsilon) d l-\int_{-\infty}^{\infty} p(m) \Theta(|m|-\epsilon) d m
\end{gathered}
$$

when we assume that the underlying stochastic process is ergodic, namely, that time average and ensemble average 
coincide. For the ordinary Wiener process, the probability distributions for $S_{t}$ and $S_{t-1}$ are Gaussians with zero mean and variances $t \sigma^{2}$ and $(t-1) \sigma^{2}$, respectively. Thus we easily evaluate the integral appearing in Eq. (6) after substituting $p=p_{G}\left(S_{t}\right)=\left(1 / \sqrt{2 \pi \sigma^{2} t}\right) e^{-S_{t}^{2} / 2 \sigma^{2} t}$ and $p_{G}\left(S_{t-1}\right)=\left[1 / \sqrt{2 \pi \sigma^{2}(t-1)}\right] e^{-S_{t-1}^{2} / 2 \sigma^{2}(t-1)}$ and obtain $P(t)$ $=\Omega\left(p_{G}: S_{t}, S_{t-1}\right)$ as

$$
P(t)=2\left\{H\left(\frac{a}{\sqrt{t}}\right)-H\left(\frac{a}{\sqrt{t-1}}\right)\right\},
$$

where we defined $a \equiv \epsilon / \sigma$. The function $H(x)$ is defined by $H(x) \equiv \int_{x}^{\infty} d z e^{-z^{2} / 2} / \sqrt{2 \pi}$. We should keep in mind that the above result is valid for discrete time $t$, however, it is easy for us to obtain its continuous time version by replacing $t$ $\rightarrow t, t-1 \rightarrow t-\Delta t$, and evaluating Eq. (7) in the limit of $\Delta t$ $\rightarrow 0$. Then, we have

$$
\begin{aligned}
P(t) \Delta t & =2 H\left(\frac{a}{\sqrt{t}}\right)-2 H\left(\frac{a}{\sqrt{t-\Delta t}}\right) \\
& =2 \frac{\partial}{\partial t} H\left(\frac{a}{\sqrt{t}}\right) \Delta t+O\left((\Delta t)^{2}\right) .
\end{aligned}
$$

Thus the FPT distribution for the ordinary Wiener process in the continuous time limit is given by

$$
P(t)=2 \frac{\partial}{\partial t} H\left(\frac{a}{\sqrt{t}}\right)=\frac{a \exp \left(-\frac{a^{2}}{2 t}\right)}{\sqrt{2 \pi} t^{3 / 2}} .
$$

This well-known form is the expected inverse Gaussian distribution [21] for the FPT distribution of the ordinary Wiener process and is often observed in the so-called interspike interval (ISI) of the integrate-and-fire model for neural networks $[3,4,22]$. Therefore in the asymptotic regime $t \rightarrow \infty$, the FPT distribution for the Wiener process obeys $t^{-3 / 2}$-scaling law. From the above discussion, we found that our approach based on direct counting of the FPT to obtain the FPT distribution is effective and gives a well-known solution for the ordinary Wiener process.

Before we move to the main section, we should comment on the much shorter derivation of the above formulation (9). Let us define our stochastic process by $x(t)$ and the probability density function finding the process in $x^{\prime}$ at time $t$ provided that it was in $x$ at time 0 by $p\left(x^{\prime}, t \mid x, 0\right)$. Here, we assume that $p\left(x^{\prime}, t \mid x, 0\right)$ is symmetric with respect to $x^{\prime}=0$. We also define the FPT with absorbing barriers $x= \pm \epsilon$ by $\tau$. Then, the complementary cumulative distribution of $\tau$ is given by

$$
W(x, t)=P_{r}(\tau \geqslant t)=\int_{-\epsilon}^{\epsilon} p\left(x^{\prime}, t \mid x, 0\right) d x^{\prime}
$$

and the cumulative distribution function leads to

$$
H(x, t)=P_{r}(\tau<t)=1-W(x, t)=2 \int_{\epsilon}^{\infty} p\left(x^{\prime}, t \mid x, 0\right) d x^{\prime} .
$$

Thus the FPT distribution is obtained by

$$
p(x, t)=\frac{\partial H(x, t)}{\partial t} .
$$

This equation coincides with Eq. (9). We should also mention that the similar derivations are found in Refs. $[23,24]$.

\section{STABLE PROCESSES AND THEIR FPT DISTRIBUTIONS}

We stress that our method is widely applicable to stochastic processes whose distributions are stable. Stable processes are specified as follows. If stochastic variables $Y_{i}(i$ $=1, \ldots, N)$ are identically independently distributed from $p\left(Y_{i}\right)$, the Fourier transform of the sum of the $Y_{i}$, namely, $S_{n}=\sum_{j=1}^{N} Y_{j}$ is given by

$$
\varphi_{n}(q)=[\varphi(q)]^{n},
$$

where $\varphi(q)$ is the Fourier transform of the stochastic variable $Y$, namely, the characteristic function and defined by

$$
\varphi(q)=\int_{-\infty}^{\infty} p(Y) e^{i q Y} d Y .
$$

Then, the stochastic process $Y_{i}$ is referred to as a stable process. Strictly speaking, Eq. (13) is a possible definition of infinitely divisible random variables and not of stable random variables. A stable random variable is infinitely divisible and stability refers to the invariance of the distribution with respect to convolutions.

It is obvious that for this class of stable processes, the FPT distribution is easily obtained by our method because the probability distributions $p\left(S_{t}\right)$ and $p\left(S_{t-1}\right)$ to evaluate $\Omega\left(p: S_{t}, S_{t-1}\right)$ in Eq. (5) are defined explicitly. In the next sections, we show several results from our approach.

\section{A. Lorentzian stochastic processes}

As a first simple example of the stable distributions, let us think about Lorentzian stochastic processes: $X_{t+1}=X_{t}+Y_{t}$, where the noise term $Y_{t}$ obeys the following white Lorentzian:

$$
p\left(Y_{t}\right)=\frac{\gamma}{\pi} \frac{1}{\gamma^{2}+Y_{t}^{2}} .
$$

Then, the characteristic function of the stochastic variable $Y_{t}$ is given by

$$
\varphi(q)=\frac{\gamma}{\pi} \int_{-\infty}^{\infty} \frac{e^{i q Y_{t}}}{\gamma^{2}+Y_{t}^{2}} d Y_{t}=e^{-\gamma|q|} .
$$

By using the convolution of the Fourier transform for the variable $S_{n}=\sum_{k=1}^{n} Y_{k}$, we have $\varphi_{n}(q)=[\varphi(q)]^{n}=e^{-n \gamma|q|}$. Therefore the inverse Fourier transform of $\varphi_{n}(q)$ leads to the prob- 
ability distribution of the sum of noise term as follows:

$$
p_{\text {Lorentz }}\left(S_{n}\right)=\frac{1}{2 \pi} \int_{-\infty}^{\infty} e^{-n \gamma|q|-i q x} d q=\frac{\gamma n}{\pi} \frac{1}{(\gamma n)^{2}+S_{n}^{2}} .
$$

By substituting this probability $p_{\text {Lorent }}\left(S_{t}\right), p_{\text {Lorentz }}\left(S_{t-1}\right)$ into Eqs. (5) and (6), we obtain the FPT distribution for the Lorentzian stochastic processes as follows:

$$
\begin{aligned}
P(t) & =\Omega\left(p_{\text {Lorentz }}: S_{t}, S_{t-1}\right) \\
& =2 \int_{\epsilon}^{\infty} \frac{\gamma t}{\pi} \frac{d S_{t}}{(\gamma t)^{2}+S_{t}^{2}}-2 \int_{\epsilon}^{\infty} \frac{\gamma(t-1)}{\pi} \frac{d S_{t-1}}{[\gamma(t-1)]^{2}+S_{t-1}^{2}} \\
& =\frac{2}{\pi} \tan ^{-1}\left(\frac{b}{t-1}\right)-\frac{2}{\pi} \tan ^{-1}\left(\frac{b}{t}\right),
\end{aligned}
$$

where we defined $b \equiv \epsilon / \gamma$. This is a result for discrete time steps; however, its continuous version is easily obtained by using $d\left[\tan ^{-1}(y)\right] / d y=\left(1+y^{2}\right)^{-1}$ as follows:

$$
P(t)=-\frac{\partial}{\partial t}\left\{\frac{2}{\pi} \tan ^{-1}\left(\frac{b}{t}\right)\right\}=\frac{2 b}{\pi} \frac{1}{b^{2}+t^{2}},
$$

From this result, we find that the FPT distribution for the Lorentzian stochastic processes obeys the Lorentzian. In the asymptotic regime $t \rightarrow \infty$, the FPT distribution for the Lorentzian stochastic processes obeys the $t^{-2}$-scaling law.

\section{B. Anomalous diffusion of Lévy flight}

We next consider the case of Lévy stochastic processes whose noise term $Y_{t}$ of the stochastic process $X_{t+1}=X_{t}+Y_{t}$ obeys the following Lévy distribution:

$$
p_{\text {Lévy }}\left(Y_{t}\right)=\frac{1}{\pi} \int_{0}^{\infty} e^{-\gamma|q|^{\alpha}} \cos \left(q Y_{t}\right) d q \text {. }
$$

We should keep in mind that the above distribution (20) is reduced to the Wiener stochastic process $(\alpha=2)$ and the Lorentzian stochastic process $(\alpha=1)$ as its special cases. As this process $Y_{t}(t=1, \ldots, n)$ is also stable, the sum of the noise term $S_{n}=\sum_{j=1}^{n} Y_{j}$ has the following probability distribution:

$$
p_{\text {Lévy }}\left(S_{n}\right)=\frac{1}{\pi} \int_{0}^{\infty} e^{-\gamma n|q|^{\alpha}} \cos \left(q S_{n}\right) d q
$$

Now, we can derive the FPT distribution by substituting $p_{\text {Lévy }}\left(S_{t}\right), p_{\text {Lévy }}\left(S_{t-1}\right)$ into Eqs. (5) and (6) as

$$
\begin{aligned}
P(t)= & \Omega\left(p_{\text {Lévy }}, S_{t}, S_{t-1}\right) \\
= & \frac{2}{\pi} \int_{\epsilon}^{\infty} d S \int_{0}^{\infty} e^{-\gamma t|q|^{\alpha}} \cos (q S) d q \\
& -\frac{2}{\pi} \int_{\epsilon}^{\infty} d S \int_{0}^{\infty} e^{-\gamma(t-1)|q|^{\alpha}} \cos (q S) d q .
\end{aligned}
$$

The expression of a continuous time version (22) is obtained from the derivative of the above discrete time distribution $P(t)$ with respect to $t$ as

$$
\begin{aligned}
P(t) & =\frac{\partial}{\partial t}\left\{\frac{2}{\pi} \int_{\epsilon}^{\infty} d S \int_{0}^{\infty} e^{-\left.\gamma t q\right|^{\alpha}} \cos (q S) d q\right\} \\
& =-\frac{2 \gamma}{\pi} \int_{\epsilon}^{\infty} d S \int_{0}^{\infty}|q|^{\alpha} e^{-\gamma t|q|^{\alpha}} \cos (q S) d q .
\end{aligned}
$$

In the asymptotic regime $t \rightarrow \infty$, by replacing the variable as $t q^{\alpha}=Q$ and after some simple algebra, we obtain

$$
\begin{aligned}
P(t) & =\frac{2 \gamma t^{-(\alpha+1) / \alpha}}{\pi \alpha} \int_{\epsilon}^{\infty} d S \int_{0}^{\infty} Q^{1 / \alpha} e^{-\gamma Q} \cos \left[\pi+\left(\frac{Q}{t}\right)^{1 / \alpha} S\right] d Q \\
& =\Psi(\alpha, \gamma) t^{-(\alpha+1) / \alpha}, \\
& \Psi(\alpha, \gamma) \equiv \frac{2 \gamma}{\pi \alpha^{2}} \sum_{l=0}^{\infty} \gamma^{-(2 l+1) / \alpha} \frac{(-1)^{l+1} \rho^{2 l+1}}{(2 l) !} \Gamma\left(\frac{2 l+1}{\alpha}\right),
\end{aligned}
$$

where the $\Gamma(x)$ means gamma function and $\rho$ is a constant of order 1 . Then, we should notice that the above scaling law is consistent with both the Wiener stochastic process $[P(t)$ $\sim t^{-3 / 2}$ for $\left.\alpha=2\right]$ and with the Lorentzian stochastic process $\left[P(t) \sim t^{-2}\right.$ for $\left.\alpha=1\right]$ we discussed in the previous sections.

As we saw in the above stochastic processes, our approach based on direct counting of the FPT is widely useful for the class of stable stochastic processes and the final expressions (5) and (6), containing at most just two integrals. Moreover, our approach can be applied to the FPT problems with a surprisingly wide variety of absorbing boundary conditions. This is one of the advantages of our method over other approaches based on analysis of Fokker-Plank equations. To show the advantage, in the next section, we apply our method to much more complicated stochastic stable process.

\section{Computer simulations}

To check the theoretical prediction of the power-law exponent for the FPT distribution, we perform computer simulations. Then, to generate the additive noise $Y_{t}$ for each time step, the Lévy stable distribution $p(Y)$ is needed. As shown by Umeno [25], the Lévy stable distribution is obtained by a superposition of a chaotic map such as

$$
Y_{n+1}=\left|\frac{1}{2}\left(\left|Y_{n}\right|^{\alpha}-\frac{1}{\left|Y_{n}\right|^{\alpha}}\right)\right|^{1 / \alpha} \operatorname{sgn}\left(Y_{n}-\frac{1}{Y_{n}}\right) \quad(0<\alpha<2),
$$

namely, for independently selected initial conditions $i$ $=1, \ldots, N$, the quantity $\sum_{i=1}^{N} Y^{(i)} / N^{1 / \alpha}$ obeys a Lévy stable law with $\alpha$, that is,

$$
p(Y)=\frac{1}{\pi} \int_{0}^{\infty} e^{-q^{\alpha}} \cos (q Y) d q .
$$



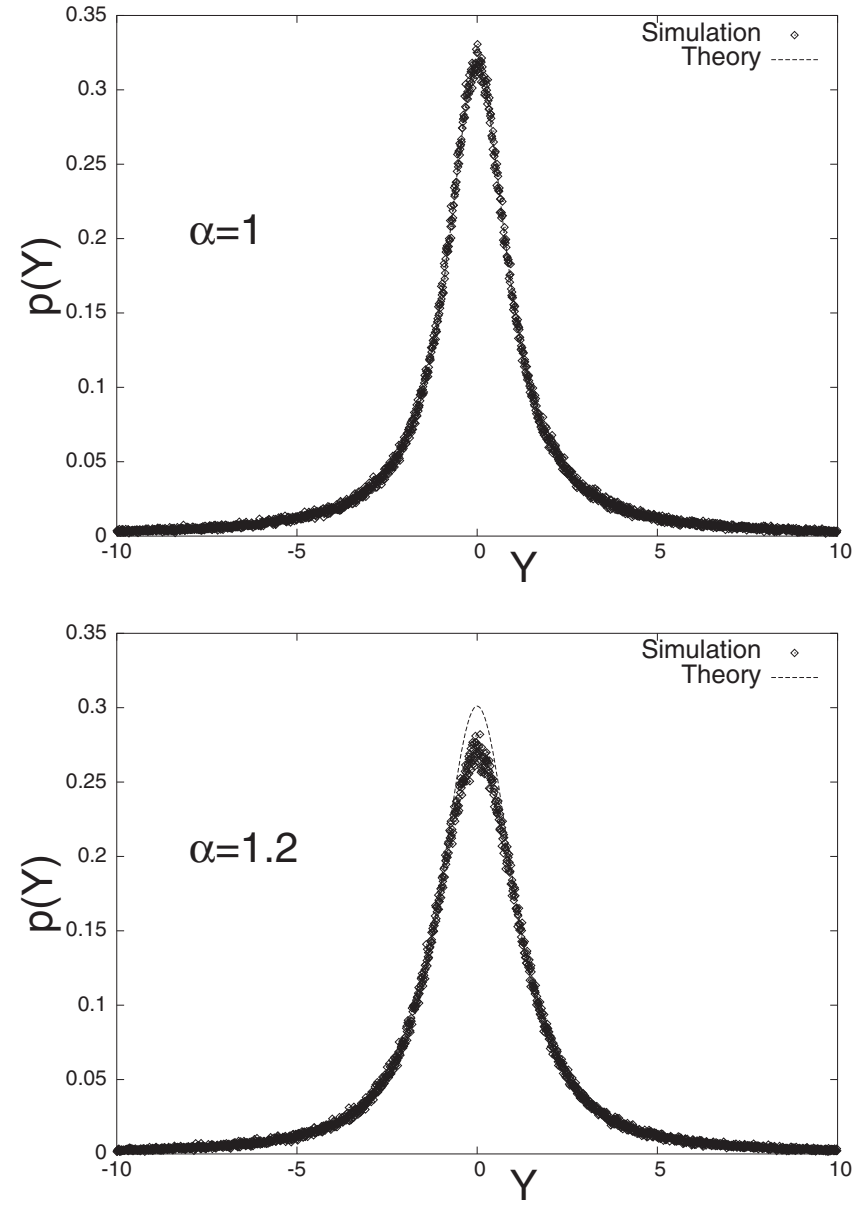

FIG. 1. Lévy distributions obtained by the chaotic map (26) with $\alpha=1$ (Lorentzian: upper) and $\alpha=1.2$ (lower). The broken lines are corresponding theoretical results given by Eq. (27). We set the number of the superposition $N=10000$.

In Fig. 1, we plot the Lévy distribution with $\alpha=1$ (Lorentzian) and $\alpha=1.2$ obtained by the superposition of the chaotic map (26). We set the number of the superposition of the chaotic map $N=10000$. From this figure, we find that the distributions obtained by the simulations are in good agreement with the corresponding analytical expressions (27). Keeping these results in mind, we use the sampling point from the superposition of the chaotic map (26) as the additive noise $Y_{t}$ in the stochastic process for each time step. Then, we should notice that one should choose a large value for the width of the rate window $\epsilon$, say, $\epsilon \sim 100$ to investigate the tail of the FPT distribution. This is because it is a rare event for the random walker to stay in the range $[-\epsilon, \epsilon]$ and it takes quite long computational time for us to obtain the tail exponent (relatively long FPT) if $\epsilon$ is small. Here, we choose $\epsilon=120$ and evaluate the power-law exponent of the FPT distribution for the Lévy processes with $\alpha=1$ and 1.2. The results are shown in Fig. 2. From this figure, we find that the power-law tails have almost the same exponents as those predicted by our theory. Of course, the tail region is too noisy to conclude that the simulations are completely consistent with the theory. However, at least we may say that they are not in disagreement. Thus the computer simulations provided us with a justification of our theoretical formulation.
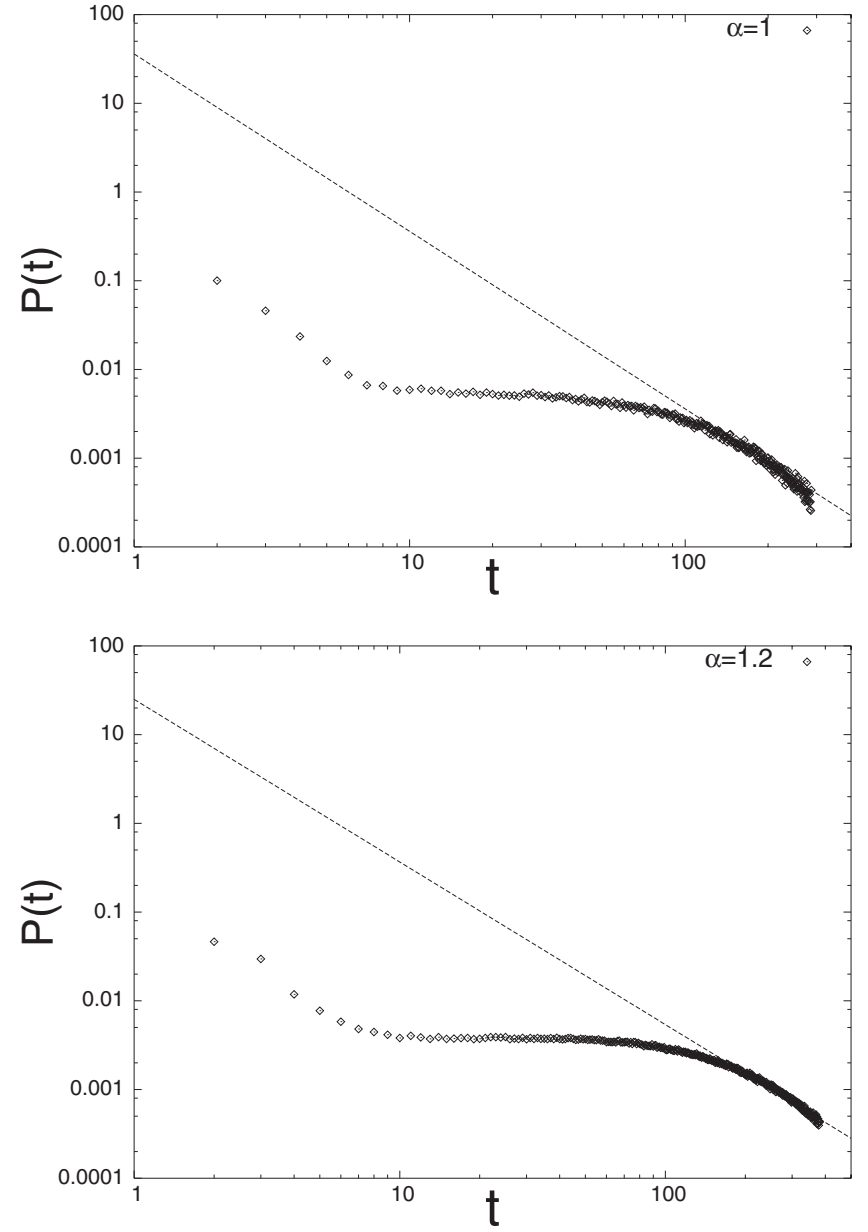

FIG. 2. The tail exponents of the FPT distributions for $\alpha=1$ (upper) and $\alpha=1.2$ (lower). The values of the power-law exponent predicted by our theory (the slope of the broken line in each panel) are -2 for $\alpha=1$ and -1.8333 for $\alpha=1.2$, respectively. We set the width of the rate window $\epsilon=120$.

\section{CROSSOVER IN SCALING LAWS OF FPT DISTRIBUTIONS}

In the previous section, we showed our formulation is effective and much more simple than the approach of the (fractal) Fokker-Plank equations [14] to obtain the FPT distribution for stable stochastic processes. We actually found that the FPT distribution of the general non-Gaussian Lévy stochastic process specified by parameter $\alpha$ is obtained and its scaling behavior in the asymptotic regime $t \rightarrow \infty$ is $t^{-(\alpha+1) / \alpha}$-law. In this section, we show that our formalism is also useful in obtaining the FPT distribution for the so-called truncated Lévy flight (the so-called KoBoL processes from Koponen, Boyarchenko, and Levendorskii [17,26,27]), for which it is well-known that the crossover between the Lévy and a Gaussian regime in the distribution of the real time step takes place [15-17,28]. In this section, we show, by using our method based on direct counting of the FPT, this kind of crossover in scaling laws is also observed in the FPT $t$.

The characteristic function for the truncated Lévy flight is defined by 


$$
T(q)=\exp \left[-\gamma \frac{\left(\Delta^{2}+|q|^{2}\right)^{\alpha / 2} \cos \left[\alpha \tan ^{-1}\left(\frac{|q|}{\Delta}\right)\right]-\Delta^{\alpha}}{\cos \left(\frac{\pi \alpha}{2}\right)}\right],
$$

where $\tan ^{-1}(|q| / \Delta) \simeq \pi / 2$ as $\Delta \rightarrow 0$ [29]. Therefore in this limit $\Delta \rightarrow 0$, Eq. (28) is reduced to $T(q)=e^{-\gamma|q|^{\alpha}}$. Obviously, this is identical to the characteristic function of the conventional Lévy flight as we already saw in the previous section. For this reason, a nonzero value of $\Delta$ controls the cutoff width of the truncated Lévy flight. We should notice that one could also use a hard cutoff version of the truncation scheme [15], namely,

$$
p_{T L F}\left(Y_{t}\right)=p_{\text {Lévy }}\left(Y_{t}\right) \Theta\left(\Delta^{-1}-\left|Y_{t}\right|\right)
$$

However, for its mathematical simplicity, we use the soft cutoff version of the truncation scheme, which will be explained below.
By the convolution of the Fourier transform, we can show

$$
\begin{aligned}
T_{n}(q) & =[T(q)]^{n} \\
& =\exp \left[-n \gamma \frac{\left(\Delta^{2}+|q|^{2}\right)^{\alpha / 2} \cos \left[\alpha \tan ^{-1}\left(\frac{|q|}{\Delta}\right)\right]-\Delta^{\alpha}}{\cos \left(\frac{\pi \alpha}{2}\right)}\right]
\end{aligned}
$$

and then the sum of the noise term $S_{n}=\sum_{j=1}^{n} Y_{j}$ of the truncated Lévy flight obeys the following probability distribution:

$$
\begin{aligned}
& p_{T L F}\left(S_{n}\right)=\frac{1}{2 \pi} \int_{-\infty}^{\infty} d q e^{-i q S_{n}}[T(q)]^{n}=\frac{1}{\pi} \int_{0}^{\infty} d q \cos \left(q S_{n}\right) \\
& \times\left[-n \gamma \frac{\left(\Delta^{2}+|q|^{2}\right)^{\alpha / 2} \cos \left[\alpha \tan ^{-1}\left(\frac{|q|}{\Delta}\right)\right]-\Delta^{\alpha}}{\cos \left(\frac{\pi \alpha}{2}\right)}\right] .
\end{aligned}
$$

Substituting these probability distributions $p_{T L F}\left(S_{t}\right)$, $p_{T L F}\left(S_{t-1}\right)$ into Eqs. (6) and (5), and taking the derivative of $P(t)$ with respect to $t$, we obtain the FPT distribution of the truncated Lévy flight for the continuous time case as

$$
\begin{aligned}
P(t)= & -\frac{2 \gamma}{\pi} \int_{\epsilon}^{\infty} d S \int_{0}^{\infty} d q\left[\frac{\left(\Delta^{2}+|q|^{2}\right)^{\alpha / 2} \cos \left[\alpha \tan ^{-1}\left(\frac{|q|}{\Delta}\right)\right]-\Delta^{\alpha}}{\cos \left(\frac{\pi \alpha}{2}\right)}\right] \\
& \times \exp \left[-\gamma t \frac{\left(\Delta^{2}+|q|^{2}\right)^{\alpha / 2} \cos \left[\alpha \tan ^{-1}\left(\frac{|q|}{\Delta}\right)\right]-\Delta^{\alpha}}{\cos \left(\frac{\pi \alpha}{2}\right)}\right] \cos (q S) .
\end{aligned}
$$

Thus far, it has been difficult to perform the above two integrals with respect to $S$ and $q$ analytically to obtain a compact form of the FPT distribution. However, numerical integrations of Eq. (32) enable us to proceed to it. In Fig. 3, we show the scaling plot of the FPT distribution for the truncated Lévy flight with $\alpha=1.2$ for several values of $\Delta$. From these three panels in Fig. 3, we find that the scaling law of the FPT distribution changes from $t^{-(\alpha+1) / \alpha}=t^{-(1.2+1) / 1.2}$ $\sim t^{-1.83}$ to $t^{-3 / 2}$ at some crossover points $t_{\times} \simeq 44(\Delta=0.5), 19$ $(\Delta=1.0)$, and $t_{\times} \simeq 8(\Delta=2.0)$. To obtain useful information about the crossover point $t_{\times}$, we evaluate the asymptotic form of the FPT distribution (32) for both $|q| \leqslant \Delta$ (Gaussian regime) and $|q|>\Delta$ (non-Gaussian Lévy regime).
For the Gaussian regime $|q| \leqslant \Delta$, by replacing the variable $q$ with $Q$ as $\gamma t \alpha^{2} \Delta^{\alpha-2} q^{2} /[2 \cos (\pi \alpha / 2)]=Q$, that is,

$$
q=\sqrt{\frac{2 Q \cos \left(\frac{\pi \alpha}{2}\right)}{\gamma \alpha^{2} \Delta^{\alpha-2}}} t^{1 / 2}
$$

we obtain

$$
P(t)=\psi(\alpha, \gamma, \Delta) t^{-3 / 2},
$$



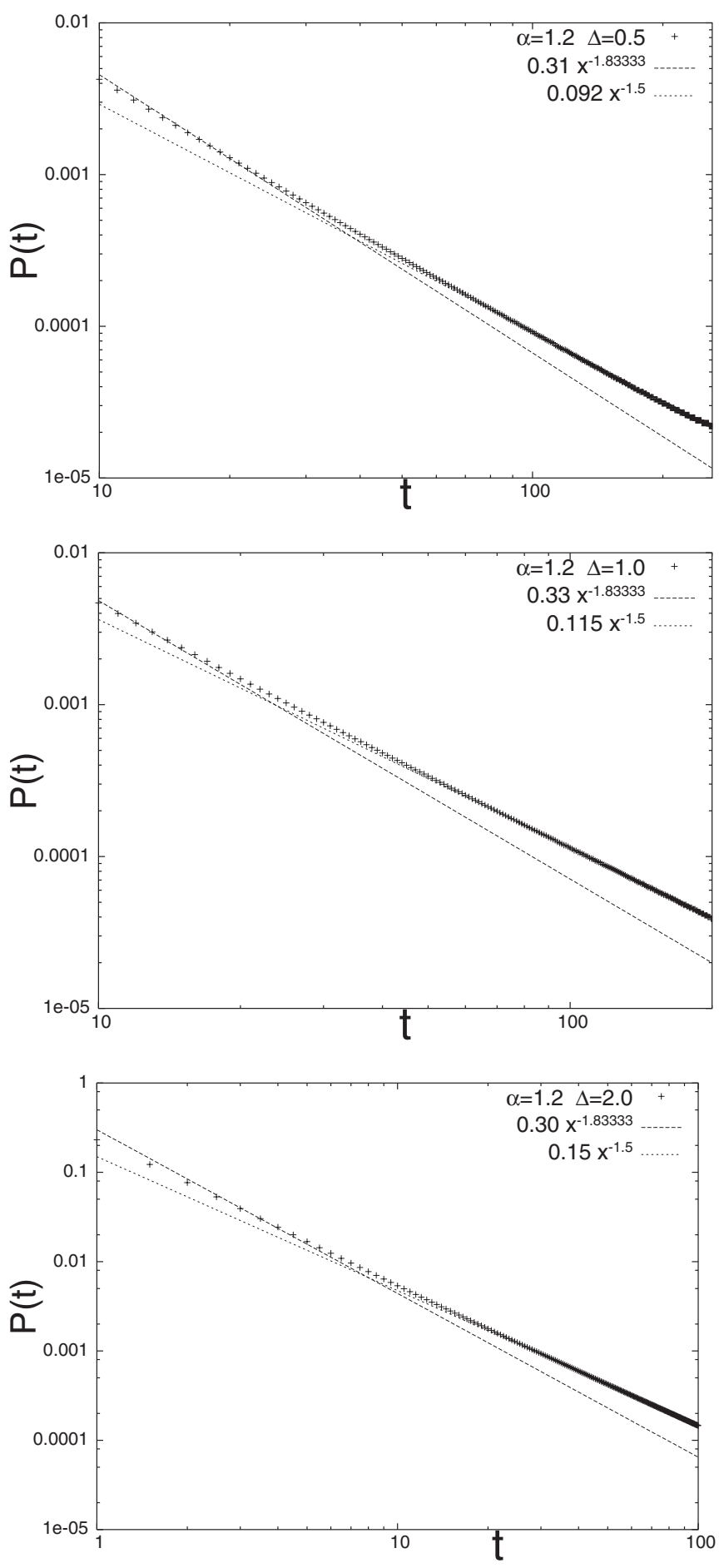

FIG. 3. Scaling laws of the FPT distribution for the truncated Lévy flight. We set $\alpha=1.2$ and $\Delta=0.5$ (upper panel), 1.0 (center panel) and 2.0 (lower panel). We find a clear crossover between Lévy and Gaussian regimes. The scaling laws change at the crossover point $t_{\times} \simeq 44(\Delta=0.5), 19(\Delta=1.0)$, and $t_{\times} \simeq 8(\Delta=2.0)$. Below the crossover point $t_{\times}$, the scaling laws are those of the Lévy: $t^{-(\alpha+1) / \alpha}=t^{-1.83}$, whereas, above $t_{\times}$, the scaling laws become those of the Gaussian: $t^{-3 / 2}$. In these three panels, we find that the scaling relation $t_{\times}(\Delta=0.5) / t_{\times}(\Delta=1.0)=t_{\times}(\Delta=1.0) / t_{\times}(\Delta=2.0)=2^{\alpha} \simeq 2.297$ holds for the crossover points.

$$
\begin{aligned}
\psi(\alpha, \gamma, \Delta) \equiv & \frac{\sqrt{2}}{\pi}\left(\frac{\gamma \alpha^{2} \Delta^{\alpha-2}}{\cos \left(\frac{\pi \alpha}{2}\right)}\right)^{-1 / 2} \int_{\epsilon}^{\infty} d S \int_{0}^{\infty} d Q Q^{1 / 2} e^{Q} \\
& \times \cos \left[\sqrt{\frac{\gamma \alpha^{2} \Delta^{\alpha-2}}{2 \cos \left(\frac{\pi \alpha}{2}\right)}} Q^{1 / 2} S\right] .
\end{aligned}
$$

It should be noted that this $t^{-3 / 2}$ law is valid for $|q| \leqslant \Delta$. From Eq. (33), this condition reads

$$
t \geqslant \frac{2 Q \cos \left(\frac{\pi \alpha}{2}\right)}{\gamma \alpha^{2} \Delta^{\alpha}} \equiv t_{\times} .
$$

On the other hand, for $|q|>\Delta$, that is to say, for $t<t_{\times}$, the FPT distribution (32) is evaluated as

$$
\begin{aligned}
P(t) \simeq & -\frac{2 \gamma}{\pi} \int_{\epsilon}^{\infty} d S \int_{0}^{\infty} d q\left[\frac{q^{\alpha}\left\{\cos \left(\frac{\pi \alpha}{2}\right)-\left(\frac{\Delta}{q}\right)^{\alpha}\right\}}{\cos \left(\frac{\pi \alpha}{2}\right)}\right] \\
& \times e^{-\gamma|q| t} \cos (q S) \simeq-\frac{2 \gamma}{\pi} \int_{\epsilon}^{\infty} d S \int_{0}^{\infty} d q|q|^{\alpha} e^{-\gamma|q|^{\alpha_{t}}} \cos (q S) .
\end{aligned}
$$

This result is identical to the FPT distribution for the conventional Lévy flight, which is defined by Eqs. (24) and (25), and was already obtained in the previous section.

Let us summarize the result for the scaling laws of the FPT distribution for the truncated Lévy flight.

$$
P(t) \sim \begin{cases}t^{-(\alpha+1) / \alpha} & \left(t<t_{\times}: \text {non-Gaussian Lévy regime }\right) \\ t^{-3 / 2} & \left(t \geqslant t_{\times}: \text {Gaussian regime }\right) .\end{cases}
$$

We should bear in mind that the crossover point $t_{\times}$obtained by Eq. (36) contains the integral variable $Q$. Therefore it is hard to say that $t_{\times}$is well-defined. To delete the $Q$-dependence of $t_{\times}$, we consider the ratio of $t_{\times}(\Delta)$ and $t_{\times}(2 \Delta)$. From Eq. (36), we obtain $t_{\times}(\Delta) / t_{\times}(2 \Delta)=2^{\alpha}$, namely, $t_{\times}(\Delta)=2^{\alpha} t_{\times}(2 \Delta)$. Let us check this scaling relation for the result we obtained in Fig. 3. For $\alpha=1.2$, the relation reads $t_{\times}(\Delta)=2^{1.2} t_{\times}(2 \Delta)=2.297 t_{\times}(2 \Delta)$. This relation predicts the crossover point $t_{\times}(\Delta=0.5) / t_{\times}(\Delta=1.0)=t_{\times}(\Delta=1.0) / t_{\times}(\Delta$ $=2.0)=2.297$, which is very close to the results obtained in Fig. 3, namely, $44 / 19 \simeq 2.316$ and $19 / 8 \simeq 2.375$. The small difference is probably because of impreciseness of numerical integrations appearing in Eq. (32).

The relation $t_{\times}(\Delta)=2^{\alpha} t_{\times}(2 \Delta)$ for successive values of $\Delta$ and $2 \Delta$ is easily extended for the relation between $\Delta$ and $\delta \Delta$ $(\delta \geqslant 1)$ as follows:

$$
t_{\times}(\Delta)=\delta^{\alpha} t_{\times}(\delta \Delta) .
$$

This scaling relation for the crossover point $t_{\times}$in the scaling laws of the FPT distribution of the truncated Lévy flight $t_{\times}$is one of the main results in this paper. From this result [Eq. 
(39)], we find that the crossover point $t_{\times}$increases rapidly as the effective cutoff length $l \equiv(\delta \Delta)^{-1}$ also increases as

$$
t_{\times}(l)=\left(\frac{l}{l_{0}}\right)^{\alpha} t_{\times}\left(l_{0}\right),
$$

where we set $l_{0} \equiv \Delta^{-1}$. Therefore we conclude that the crossover between non-Gaussian Lévy and Gaussian regimes is observed not only in the distribution of the real-time $n$ flight, which was reported by Mantegna and Stanley [15], but also in the FPT distribution of the truncated Lévy flight.

In the study by Mantegna and Stanley [15], they investigated the stochastic variable $z_{n}=\sum_{k=1}^{n} x_{k}$, where $x_{k} \equiv X_{k}$ $-X_{k-1}$ obeys the truncated Lévy flight. They evaluated the probability of return $P\left(z_{n}=0\right)$ and found that the $P\left(z_{n}=0\right)$ obeys the Gaussian $n^{-1 / 2}$ law in the large real time step $n$ regime. In this section, it was shown that this ultraslow convergence from the Lévy regime to the Gaussian regime is conserved even if we consider the first passage process of the truncated Lévy flight. The relation between their results and ours is clearly understood as follows.

For a given time interval $t$ of the first passage process of the truncated Lévy flight, the time series of the variable $x_{k}$ $=X_{k_{0}+k}-X_{k_{0}+k-1}$ behaves as $x_{1}, x_{2}, \ldots, x_{t}$, where $k_{0}$ is an origin for the measurement of the interval $t$. Then, from the observation by Mantegna and Stanley, the sum $z_{t}=\sum_{k=1}^{t} x_{k}$ $=X_{k_{0}+t}-X_{k_{0}}$ obeys a Gaussian with zero-mean and variance $t$ if the time interval $t$ is large enough, that is, $t \geqslant t_{\times}$. Then, the probability of return is given by $P\left(z_{t}=0\right) \simeq t^{-1 / 2}$. In other words, for $t \geqslant t_{\times}$, it takes quite a long time for a random walker to escape from the region $\left[X_{k_{0}}-\epsilon, X_{k_{0}}+\epsilon\right]$, and the time $t$ for escaping guarantees that the central limit theorem works to make the variable $z_{t}$ a Gaussian. As a result, the FPT distribution $P(t)$ should follow the corresponding Gaussian $t^{-3 / 2}$-law from our argument for the case of the Wiener process (9). On the other hand, if the interval $t$ is smaller than the crossover point $t_{\times}$, the central limit theorem for $z_{t}$ does not work and $z_{t}$ is no longer a Gaussian. Then, as we checked, the FPT distribution $P(t)$ obeys $t^{-(\alpha+1) / \alpha}$-law of the Lévy flight.

\section{SUMMARY}

In this paper, we proposed an approach to evaluate the FPT distribution. Our method is based on direct counting of the FPT. We show that our approach gives an explicit form of the FPT distribution for stable stochastic processes. Actually, for Wiener (Brownian motion), Lorentzian, and Lévy stochastic processes, our method was demonstrated. Thanks to the mathematical simplicity of our method, it becomes easy to grasp the intuitive meaning of the FPT distribution and to tackle more complicated stochastic processes. As an example, we discussed the FPT distribution of the truncated Lévy flight (the KoBoL process). We found a clear crossover between non-Gaussian Lévy and Gaussian regimes in the scaling laws of the FPT distribution. We found the scaling relation on the crossover point $t_{\times}$with respect to the effective length $l$ of the cutoff as $t_{\times}(l)=\left(l / l_{0}\right)^{\alpha} t_{\times}\left(l_{0}\right)$ with $l_{0}$ $=\Delta^{-1}$.

Very recently, Koren et al. [30] investigated not only the FPT distribution but also the first-passage leapover (FPL) distribution under a single absorbing boundary condition. A relatively new concept, the FPL is defined as the flight length for a random walker to move beyond the single boundary (a target). Our system in this paper possesses two boundaries (in this sense, our process might be referred to as a first exit process); however, it might be possible to apply our analysis to the problem in order to discuss the FPL distribution. This will be addressed in future work.

We hope that beyond the present analysis for the Sony Bank rate, our approach might be widely used in many scientific research fields, especially in the field of econophysics including financial data analysis.

\section{ACKNOWLEDGMENTS}

One of the authors (J.I.) was financially supported by Grant-in-Aid for Young Scientists (B) of The Ministry of Education, Culture, Sports, Science and Technology (MEXT) No. 15740229 and Grant-in-Aid Scientific Research on Priority Areas "Deepening and Expansion of Statistical Mechanical Informatics (DEX-SMI)" of The Ministry of Education, Culture, Sports, Science and Technology (MEXT) No. 18079001. N.S. would like to thank Shigeru Ishi, President of the Sony Bank, for useful discussions. The authors thank the anonymous referee for many instructive comments on the paper. We also thank Enrico Scalas for fruitful discussion and useful comments fruitful discussions and useful comments.
[1] S. Redner, A Guide to First-Passage Processes (Cambridge University Press, Cambridge, England, 2001).

[2] N. G. van Kappen, Stochastic Processes in Physics and Chemistry (North-Holland, Amsterdam, 1992).

[3] H. C. Tuckwell, Introduction to Theoretical Neurobiology (Cambridge University Press, Cambridge, England, 1988), Vol. 2.

[4] H. C. Tuckwell, Stochastic Processes in the Neurosciences (Society for Industrial and Applied Mathematics, Philadelphia, 1989).
[5] I. Simonsen, M. H. Jensen, and A. Johansen, Eur. Phys. J. B 27, 583 (2002).

[6] M. Raberto, E. Scalas, and F. Mainardi, Physica A 314, 749 (2002).

[7] E. Scalas, R. Gorenflo, H. Luckock, F. Mainardi, M. Mantelli, and M. Raberto, Quant. Finance 4, 695 (2004).

[8] S. Kurihara, T. Mizuno, H. Takayasu, and M. Takayasu, The Application of Econophysics, edited by H. Takayasu (Springer, New York, 2003), pp. 169-173.

[9] T. Kaizoji and M. Kaizoji, Physica A 336, 563 (2004). 
[10] E. Scalas, Physica A 362, 225 (2006).

[11] N. Sazuka, Eur. Phys. J. B 50, 129 (2006).

[12] N. Sazuka, Physica A 376, 500 (2007).

[13] http://moneykit.net/

[14] G. Rangarajan and M. Ding, Phys. Rev. E 62, 120 (2000).

[15] R. N. Mantegna and H. E. Stanley, Phys. Rev. Lett. 73, 2946 (1994).

[16] R. N. Mantegna, Phys. Rev. E 49, 4677 (1994).

[17] I. Koponen, Phys. Rev. E 52, 1197 (1995).

[18] G. Rangarajan and M. Ding, Phys. Lett. A 273, 322 (2000).

[19] G. Rangarajan and M. Ding, Fractals 8, 139 (2000).

[20] R. Durrett, Essentials of Stochastic Processes (SpringerVerlag, New York, 1999).

[21] R. Chikara and L. Folks, The Inverse Gaussian Density (Dekker, New York, 1989).

[22] W. Gerstner and W. Kistler, Spiking Neuron Models (Cam- bridge University Press, Cambridge, England, 2002).

[23] H. Risken, The Fokker-Plank Equation: Methods of Solution and Applications (Springer-Verlag, Berlin, 1989).

[24] C. W. Gardiner, Handbook of Stochastic Methods (SpringerVerlag, Berlin, 1983).

[25] K. Umeno, Phys. Rev. E 58, 2644 (1998).

[26] W. Schoutens, Lévy Processes in Finance: Pricing Financial Derivatives (Wiley, New York, 2003).

[27] S. I. Boyarchenko and S. Z. Levendorskii (unpublished).

[28] R. N. Mantegna and H. E. Stanley, An Introduction to Econophysics: Correlations and Complexity in Finance (Cambridge University Press, Cambridge, England, 2000).

[29] J. Voit, The Statistical Mechanics of Financial Markets (Springer, New York, 2001).

[30] T. Koren, A. V. Chechkin, and J. Klafter, Physica A 379, 10 (2007). 